\title{
Prediction system based on domotic weather sensors for the energy production of solar power plants
}

\author{
Domingo Benítez, Carlos González-Muñoz, José F. Medina \\ Universidad de Las Palmas de Gran Canaria \\ SIANI University Institute \\ Campus of Tafira, 35017 Las Palmas de Gran Canaria (Spain) \\ Phone/Fax number:+0034 928 458711, e-mail: dbenitez@siani.es, cgonzalez@dis.ulpgc.es, jmedina@die.ulpgc.es
}

\begin{abstract}
The prediction of the electrical energy generated by a photovoltaic system is useful for estimating the profitability analysis of a project, without the need of expensive photovoltaic prototypes. Prediction systems are usually based on simulating the physical process of a photovoltaic module, under standard or average local weather conditions. These predictions introduce some errors caused by the use of a theoretical model or average climate data.

In our investigations, we noted that the energy generated by a photovoltaic system is proportional to the cumulative measurement of the sun illuminance that is provided by a low-cost domotic weather station. From this experimental observation, this paper proposes a hardware/software system for predicting the electrical energy generated by a photovoltaic system, such as those existing in buildings. The hardware consists of a domotic installation for monitoring both electric energy and climate parameters. The software consists of a calibration procedure, which provides a proportional factor between sun illuminance and the energy production per unit of surface area of the photovoltaic modules. Once the calibration procedure is completed, the photovoltaic energy production is predicted by factoring the sun illuminance provided by the weather station and the proportional factor provided by the calibration process. This method has been tested under real conditions and the accuracy reached up to $99.7 \%$ with an average value of $96.3 \%$.
\end{abstract}

\section{Keywords}

Domotics, Energy prediction and forecasting, Sun energy, Power system operation, Smart buildings.

\section{Introduction}

Environmental concerns and the future shortage of fossil fuels keep interest in renewable energy sources. Nowadays, solar energy is a rapidly growing renewable energy source. Solar cells directly convert sunlight into electricity, with no noise and no air pollution, reducing the need for conventional fossil fuels. Photovoltaic grid connected systems arise as a result in the development of photovoltaic systems. Utility-scale photovoltaic power plants of several MW and building integrated photovoltaic systems in the range of $\mathrm{kW}$ are the two most usual ways for generating electricity from sun [2].

Domotic equipment consists of a set of computing elements that are distributed within the entire building. These elements are linked by one or several communication protocols, such as KNX, for data transmitting [3].

Many manufacturers of low voltage electrical equipment commercialize domotic devices. Among others, low-cost domotic weather sensors can be found. They also offer devices for connecting the domotic devices to a data network, in addition to metering devices for electric power and energy.

The development of domotic techniques for automation and metering of a building is an activity which grows in parallel with building integrated photovoltaic systems. Although home automation was initially focused on getting comfort and security [1], its techniques can also address to get energy efficiency.

A photovoltaic power plant and a domotic system can both coexist in a building, but they do not use to interact directly with each other [4]. A domotic system for recording the sun illuminance can be useful in estimating the power and energy production from a photovoltaic (PV) plant. That information can be transmitted through the building domotic system to the Operation and Control Centre of the bulk electric power system in that area.

An Electric Control Centre usually gets data from the actual state of loads, power transmission lines and conventional power plants. It also gets power production data from medium to large-size wind and solar power plants. The power system operator makes use of these data for performing tasks like State Estimation, Contingency Analysis, Unit Commitment and Economic Dispatch. 
Nevertheless, nowadays data received by the Electric Control Centre do not include the power generated by power plants that are integrated into buildings [2]. This could cause an error in evaluating the total amount of power generated by the whole electric system, because that kind of distributed generation should not be currently considered negligible. Domotic systems can provide a solution to this emerging problem $[4,5]$.

In this paper, we propose a hardware/software system based on domotic weather sensors for estimating the energy production of a photovoltaic plant that is integrated into a building. We have used a real solar plant and a real domotic installation to measure the accuracy of the power and energy prediction that is provided by our system.

\section{Experimental Methodology}

For achieving our objectives, we use a domotic KNX installation for metering the energy and power production that is supplied by a $20 \mathrm{~kW}$ photovoltaic plant (see Figure 1). In this paper, we use the values measured during seven days in March 2010 (from 23 $3^{\text {th }}$ to $29^{\text {th }}$ ). The installation includes a domotic weather station (see Figure 2), which monitors the sun illuminance in Lux.

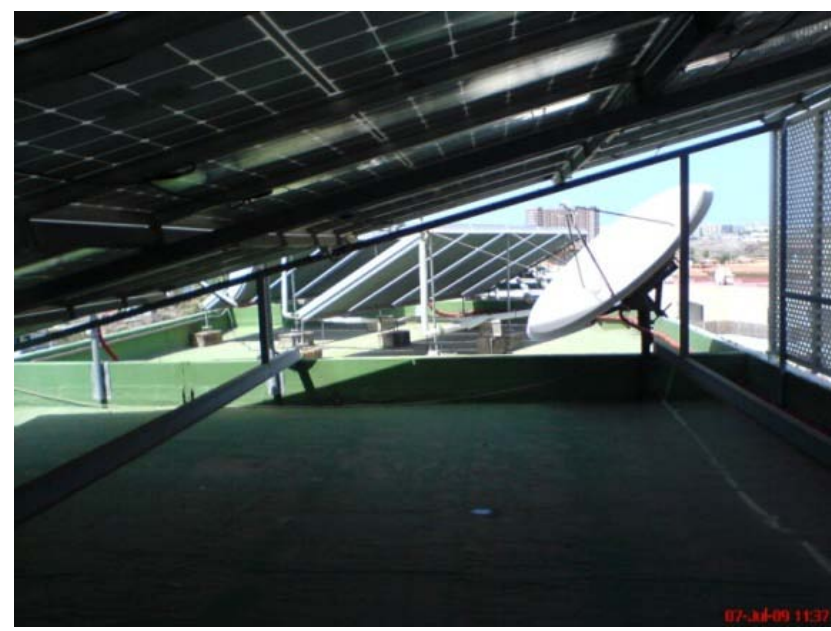

Fig. 1. Photovoltaic power plant

The PV plant consists of solar modules with an area of $176 \mathrm{~m}^{2}$ that is placed on the roof of a building, which is oriented to the South. A three phase inverter is used to convert the DC into AC current, which is injected to the low voltage electric grid. The connection of the metering equipment to the AC power lines is made with three current transformers. The electronic meter is also connected to a domotic KNX bus that allows us to get monitoring data, which are sent to a computer.

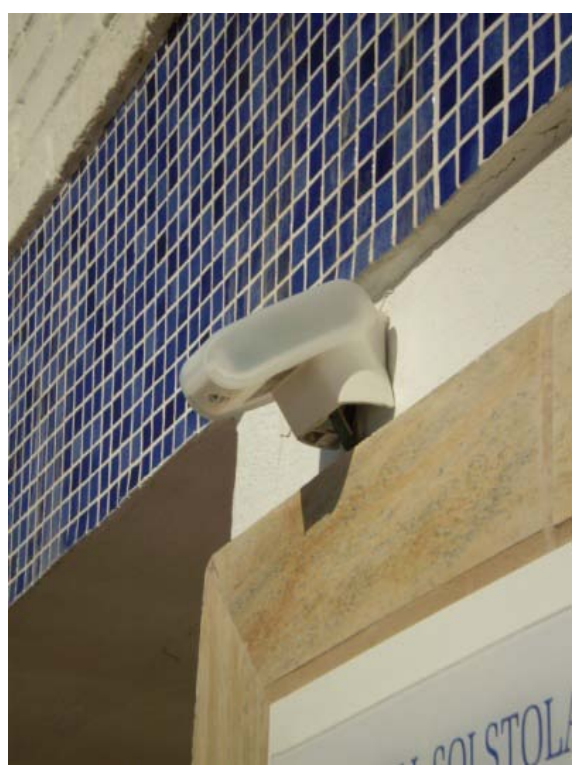

Fig. 2. Domotic weather station

In our experiments, we registered the power and energy that was generated by the PV plant that is shown in Figure 1. The data obtained for PV power during one of the days involved in the experiments can be seen in Figure 3. Additionally, we synchronously registered the sun illuminance during 24-hour intervals, by means of the domotic weather station that is shown in Figure 2. The data obtained for the sun illuminance during the same day that was used in Figure 3 can be seen in Figure 4 . Note that this curve shows a long plateau during the central hours of the day with different time intervals of illuminance fall due to clouds.

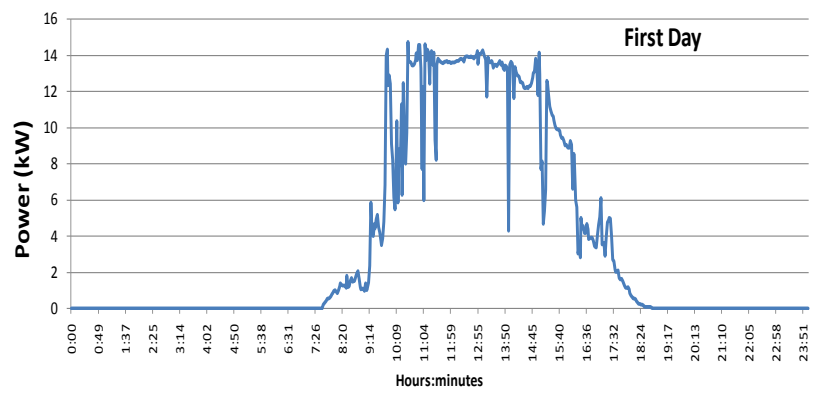

Fig. 3. Power generated by the PV plant for the first day of the week that was involved in the experiments.

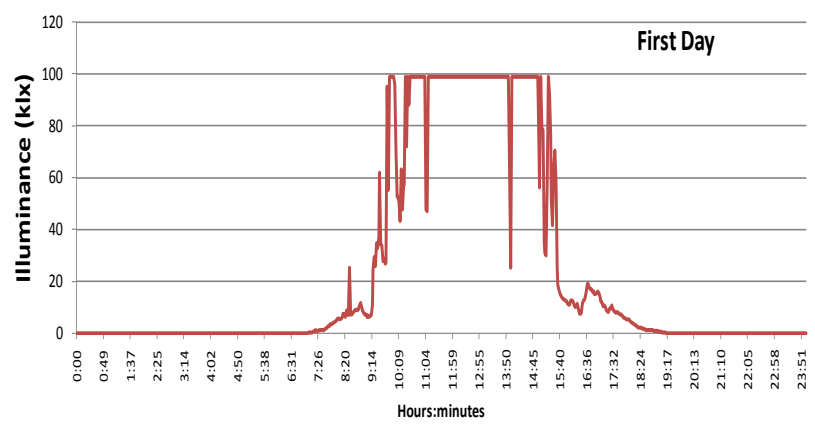

Fig. 4. Illuminance of the solar radiation for the first day of the week that was involved in the experiments. 
The correlation between the power and illuminance curves can be clearly seen in Figure 5, where both curves are overlaid. Note the matching of both curves. The variability of solar radiation due to clouds in the center hours of the day follows the power curve.

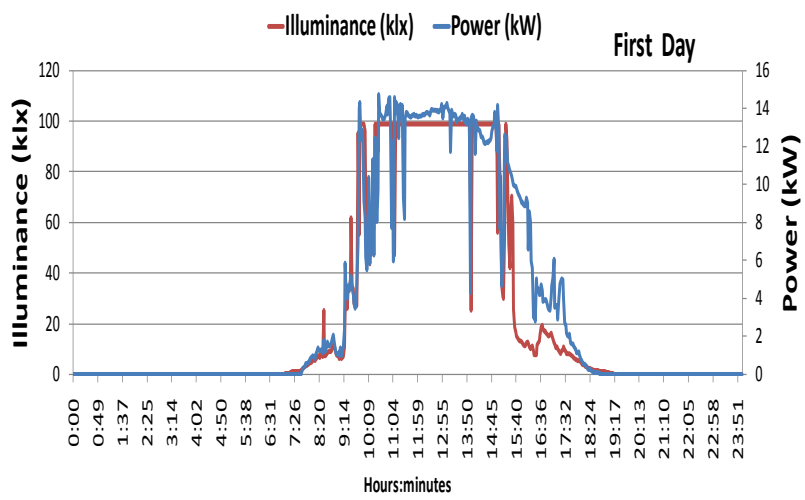

Fig. 5. Illuminance of the solar radiation versus power generated by the PV plant for the first day of the week that was involved in the experiments.

In a sunny day, it is possible to clearly observe the close relationship between the power and sun illuminance curves. Figure 6 shows the data that we registered during the third day of the week that was involved in our experiments. The PV energy that was generated during this day was the highest energy obtained for one complete day.

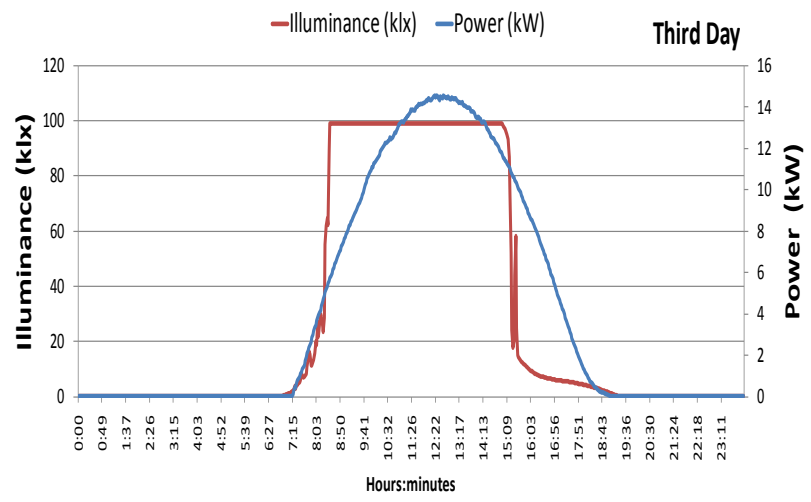

Fig. 6. Sun illuminance versus power generated by the PV plant for the third day of the week that was involved in the experiments.

Note in Figure 6 the shape of both curves, which can be representatives of a day without clouds. The illuminance curve is flat during the central hours of the day (from 8:15 to $15: 15$ approximately). This flat shape is caused by the saturation of the illuminance sensor that is integrated into the domotic weather station. However, the power curve is not saturated and follows the position angle of the sun with respect to the perpendicular axis of the PV modules. When the power curve has reached the maximum, the deviation of the position angle of the sun from the perpen- dicular axis of the PV modules is lower than in other hours of the day.

If the principal and secondary vertical axes of Figure 6 are normalized by using the respective maximum values of each axis, we observed that the areas under both curves are approximately equal. The area that is under the section of the illuminance curve over the power curve during the early hours of the day is balanced with the respective areas during the midday and evening hours.

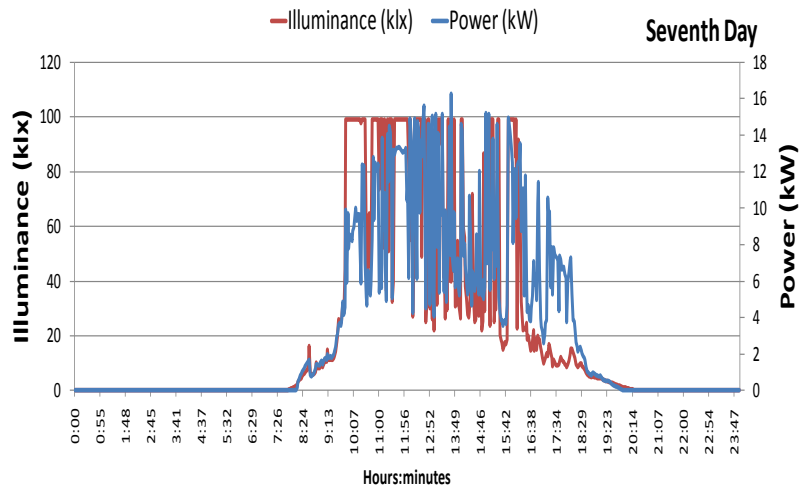

Fig. 7. Sun illuminance versus power generated by the PV plant for the seventh day of the week that was involved in the experiments.

From the point of view of energy production, the seventh day was the worst day. This was caused by the amount and density of cloud cover during that day. Figure 7 shows the power and sun illuminance curves that were registered with our domotic KNX installation. The shapes of both curves are influenced by the cloudy weather conditions during the seventh day. Note that the illuminance curve closely follows the power curve when the illuminance sensor is not saturated.

The energy production can be measured by integrating the power curve. Thus, when the energy production for a day is lower than for another day, the area under the power curve for the first day is smaller than the area under the power curve for the second day. Since we have observed that the area under the sun illuminance curve is proportional to the area under the power curve, our conjecture is that with the area under the illuminance curve, the photovoltaic energy production can be modelled and predicted.

From all these experimental results, our goal was to propose a theoretical method for predicting the energy production of PV plants by using low-cost domotic weather sensors. The next section describes a simple modeling approach for the photovoltaic energy production that can be easily implemented in a computer. 


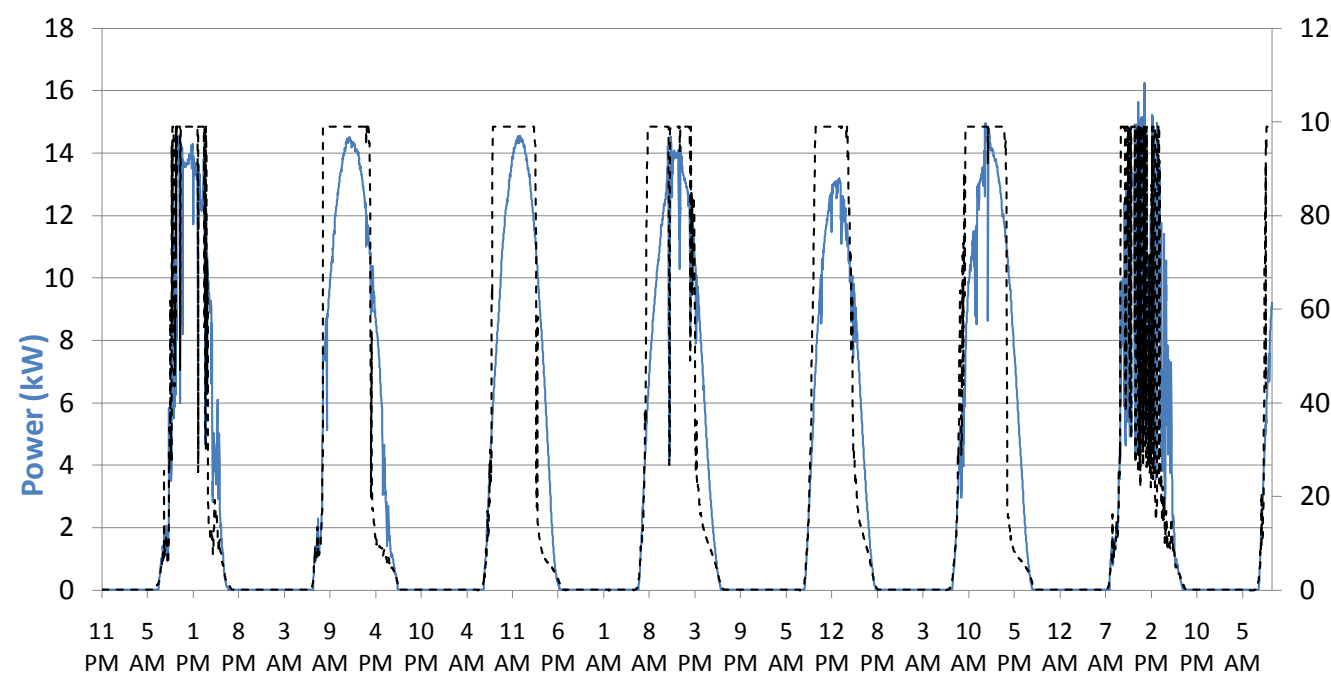

PM AM PM PM AM AM PM PM AM AM PM AM AM PM PM AM PM PM AM AM PM AM AM PM PM AM

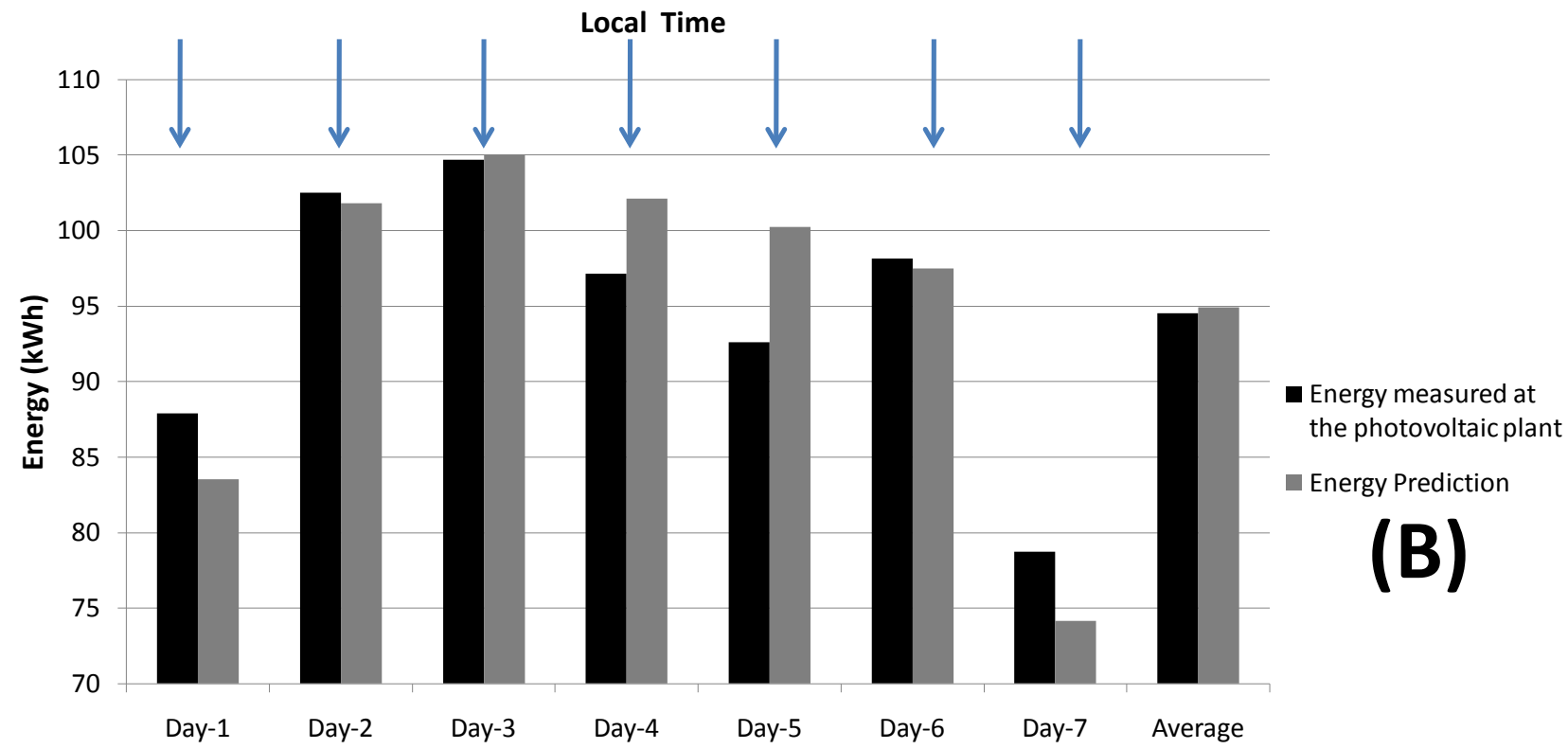

Fig. 8. (A) Sun illuminance (klx) and electric power production $(\mathrm{kW})$ at the photovoltaic power plant. (B) Energy production (kWh) that was measured at the photovoltaic plant versus our photovoltaic energy prediction (kWh).

\section{Modelling Approach}

The system proposed for predicting the energy that could be produced by a PV power plant is based on the experimental results that were described above. These observations suggest a proportional relationship between (a) the energy $E$ produced by a PV system in a given day and (b) the amount of sun illuminance $L$ received by the PV modules during the curse of the day. The proportionality factor $\alpha$ is derived trough a procedure that we called "Calibration", which will be described later in this section.

$$
E \cong \alpha \cdot L
$$

The amount of sun illuminance received by the PV modules in the curse of a day can be estimated from the equation (2).

$$
L=\int_{t} l d t
$$

The illuminance data provided by a domotic weather station are measured periodically at sampling intervals. Therefore, illuminance can be approximately estimated as in equation (3).

$$
L \cong \sum_{t=00: 00}^{t=23: 59} l_{i} \Delta t
$$

where $l_{i}$ is the illuminance value provided by the domotic weather station at a given time and $\Delta t$ is the value of the sampling interval.

In this calibration procedure, several days must be selected for achieving an acceptable value of the proportionality factor. The factor $\alpha_{j}$ between the energy $E_{j}$ produced in a day $j$ and the corresponding amount of illumin- 
ance $L_{j}$ is calculated for every selected day according to the equation (4).

$$
\alpha_{j}=\frac{E_{j}}{L_{j}}, \forall j=1,2, \ldots, N
$$

where $N$ is the number of days used to perform the calibration procedure.

Finally, the proportionality factor $\alpha$ that can be used to predict the energy generated by the PV system is calculated as the average of the $\alpha_{j}$ factors.

$$
\alpha=\frac{1}{N} \sum_{j=1, \ldots, N} \alpha_{j}
$$

Therefore, once the factor $\alpha$ for a given location is known, our system can predict the energy that would be produced by a PV power plant by only using a low-cost domotic weather station. So, a prototype of the PV power plant is not required. In the next section, we describe the accuracy achieved by our prediction system operating under real conditions.

\section{Results}

In this section we show some results that were provided by the modeling approach for the energy production when it is applied to the real photovoltaic power plant that was described in Section 2. For this purpose, we use a time interval of seven complete days in March 2010, from $23^{\text {th }}$, to $29^{\text {th }}$.

For each one of the days used in the experiments, three measurements were registered by a domotic KNX installation [3]. Two of the measurements are electric: the energy that is supplied by the photovoltaic plant, and the RMS three-phase power. These measurements were registered by an electronic meter used for electricity metering from ABB (model DELTAplus). This meter is connected to the KNX domotic installation by using the respective interface. The electricity meter monitors the electric line that transports the three phase voltage, which is supplied by the photovoltaic DC/AC inverter circuit. The electric connection between the electricity meter and the output inverter electric lines is implemented with three current transformers. The electricity meter accumulates and saves the photovoltaic energy in its internal electronic memory. Additionally, the electricity meter registers and stores in its internal memory the photovoltaic power. We use a computer that is connected to the domotic KNX installation for directly accessing to the meter memory. In this way, the photovoltaic energy and RMS power that were saved by the electricity meter can be saved in the hard disk of the computer.

On the other hand, the outside illuminance was registered with a weather station from Siemens (model AP257/21). This module, the electricity meter and computer are connected to the same KNX domotic installation. The computer accesses to the weather station to take the sun illuminance.
In the experimental methodology, each one of the days was divided into sampling intervals, in which three measurements were taken: photovoltaic energy, photovoltaic power and sun illuminance. The sampling interval was 2.1 minutes on average. Thus, more than 600 measurements were taken each day.

With the data that were registered during seven days, we calibrated our energy prediction hardware/software system following the method that is described in Section 3. Table I shows the values of $\alpha_{i}$ that were obtained for each day when equation 4 is applied. Its average value provides the constant $\alpha(0.143)$ that appears in equation 1 . With this constant and calculating the total sun illuminance $(\mathrm{L})$ of each day using equation 3 , equation 1 provides a prediction of the energy that is supplied by the photovoltaic plant.

Table I.- Experimental data that were measured at the real photovoltaic plant, and results of the calibration method as described in Section 3.

\begin{tabular}{cccccc}
\multicolumn{7}{c}{$\begin{array}{c}\text { Energy } \\
\text { measured at }\end{array}$} \\
\multicolumn{5}{c}{$\begin{array}{c}\text { Sun illumi- } \\
\text { nance (klx), voltaic plant } \\
\text { ne }\end{array}$} & \multicolumn{3}{c}{$\begin{array}{c}\text { Energy } \\
\text { Prediction, }\end{array}$} \\
DAY & L & $\alpha_{i}$ & E (kWh) & Precision \\
\hline 1 & 15981,78 & 87,89 & 0,150 & 83,53 & $95,05 \%$ \\
2 & 22134,33 & 102,52 & 0,144 & 101,83 & $99,33 \%$ \\
3 & 20394,67 & 104,70 & 0,143 & 104,99 & $99,72 \%$ \\
4 & 22127,99 & 97,14 & 0,136 & 102,12 & $94,86 \%$ \\
5 & 18541,49 & 92,62 & 0,132 & 100,26 & $91,75 \%$ \\
6 & 20788,86 & 98,16 & 0,144 & 97,49 & $99,32 \%$ \\
7 & 13937,97 & 78,75 & 0,152 & 74,14 & $94,15 \%$ \\
\hline Average & & & 0,143 & & $96,31 \%$
\end{tabular}

Figure 8B shows results of the predicted and measured photovoltaic energy (grey and black bars respectively). The numerical results are also shown in Table I. Additionally, Figure 8A shows the power RMS and sun illuminance ( $\mathrm{Li}$ ) that were measured each sampling interval of the seven days.

As can be seen in Figure 8B and Table I, the average error of the predicted energy production is $3.7 \%$ in the time interval of seven days that was used in the experiments (see Average bars in Figure 8B and the last column of the Table I). The maximum error was $8.25 \%$ (fifth day), and the minimum error was $0.28 \%$ (third day).

These results allow us to corroborate that registering the sun illuminance with a domotic weather station can estimate with high precision the energy that is supplied by a photovoltaic plant during each day. Additionally, our proposal of calibration process is computationally simple, and its errors can be tolerated when estimating the economic profits of a photovoltaic plant. 


\section{Conclusions}

A low-cost hardware/software system that is based on domotic weather sensors has been designed for metering and predicting the power and energy production supplied by a photovoltaic power plant that is integrated into a building. A prototype system has been installed and tested under real conditions in a hotel located at a tourist area in Gran Canaria (Canary Islands, Spain).

Measurements of sun illuminance and photovoltaic electricity have been taken during a long period of time. The analysis of the system operation and the recorded measurements allow us to draw the following conclusions:

- It is possible to build a low-cost system for photovoltaic power and energy prediction using standard domotic devices. The price of our prototype is $1500 €$, which is negligible when is considering the cost of a photovoltaic plant: $5000 € / \mathrm{kWh}$.

- Our analysis shows that there exists a correlation between the sun light level that is measured by a domotic weather station and the electric power that is supplied by the photovoltaic plant. We have proposed and designed a calibration method that predicts the energy supplied by a photovoltaic plant. The precision of our prediction method is $96.3 \%$.

An application of our prediction system could be the estimation of the solar potential at a given geographic location. This evaluation of the solar energy could be useful in the design stage of a new photovoltaic plant, since a accurate and reliable preliminary profitability study can be addressed [5].

\section{Acknowledgements}

The authors acknowledge to Mr. Gustav Dahl and Mr. Carlos Hernández López for their encouragement, helpful comments and technical support. The scientific work of this paper was supported by the Spanish project called PIVE from the Cátedra-Telefónica at the University of Las Palmas de Gran Canaria.

\section{References}

[1] I. Dounis, C. Caraiscos, Advanced control systems engineering for energy and comfort management in a building environment - A review, Renewable and Sustainable Energy Reviews, Volume 13, Issues 6-7, August- September 2009, Pages 12461261

[2] J. Figueiredo, J. Martins, Energy Production System Management - Renewable energy power supply integration with Building Automation System, Energy Conversion and Management, Volume 51, Issue 6, June 2010, Pages 1120-1126

[3] KNX Standard for Buildings and Homes; www.KNX.org

[4] T. Lam Ngan Tung, Solar radiation and daylight illuminance modelling and implications for building integrated photovoltaic system designs, P.D. Thesis, City University of Hong Kong, 2008

[5] A. Muñoz-Jiménez, P. Zorzano-Santamaría, L. A. FernándezJiménez, E. Zorzano-Alba, A. Yanguas-Peña, and E. GarcíaGarrido; Short-term forecasting models for PV systems, Proceedings of the 11th CHLIE, Zaragoza, Spain. 2009 